\title{
Modulation of local field potentials by high-frequency stimulation of afferent axons in the hippocampal CA1 region
}

\author{
Ying $\mathrm{Yu}^{*}$, Zhouyan Feng**, Jiayue Cao*, Zheshan Guo*, Zhaoxiang Wang*, \\ $\mathrm{Na} \mathrm{Hu}$ and Xuefeng Wei ${ }^{\dagger}$ \\ *Ministry of Education Key Lab of Biomedical Engineering \\ College of Biomedical Engineering $\mathcal{B}$ Instrument Science \\ Zhejiang University, Hangzhou, Zhejiang 310027, P. R. China \\ Department of Biomedical Engineering \\ The College of New Jersey \\ Ewing NJ 08628, USA \\ tengzhouyan@139.com
}

[Received 22 April 2015; Accepted 27 July 2015; Published 21 October 2015]

Modulation of the rhythmic activity of local field potentials (LFP) in neuronal networks could be a mechanism of deep brain stimulation (DBS). However, exact changes of LFP during the periods of high-frequency stimulation (HFS) of DBS are unclear because of the interference of dense stimulation artifacts with high amplitudes. In the present study, we investigated LFP changes induced by HFS of afferent axons in the hippocampal CA1 region of urethaneanesthetized rats by using a proper algorithm of artifact removal. Afterward, the LFP changes in the frequency bands of $\delta, \theta, \alpha, \beta$ and $\gamma$ rhythms were studied by power spectrum analysis and coherence analysis for the recorded signals collected in the pyramidal layer and in the stratum radiatum of CA1 region before, during and after 1-min long 100 and $200 \mathrm{~Hz} \mathrm{HFS}$. Results showed that the power of LFP rhythms in higher-frequency band ( $\gamma$ rhythm) increased in the pyramidal layer and the power of LFP rhythms in lower-frequency bands $(\theta, \alpha$ and $\beta$ rhythms) decreased in the stratum radiatum during HFS. The synchronization of $\theta$ rhythm decreased and the synchronization of $\gamma$ rhythm increased during HFS in the stratum radiatum. These results suggest that axonal HFS could modulate LFP rhythms in the downstream brain areas with a plausible underlying mechanism of partial axonal blockage induced by HFS. The study provides new evidence to support the mechanism of DBS modulating rhythmic activity of neuronal populations.

Keywords: High-frequency stimulation; local field potential; artifact removal; power spectrum density; synchronization; rhythm modulation.

\section{Introduction}

Deep brain stimulation (DBS) has been investigated widely in the treatment of various neurological disorders such as Parkinson's disease, epilepsy and Alzheimer's

This is an Open Access article published by World Scientific Publishing Company. It is distributed under the terms of the Creative Commons Attribution 4.0 (CC-BY) License. Further distribution of this work is permitted, provided the original work is properly cited. 
disease (AD) (Boon et al., 2007; Hescham et al., 2013; McGovern et al., 2013). However, the underlying mechanisms of DBS are not completely clear. Many previous studies have focused on the changes of action potential firings of targeted neurons that were induced by high-frequency stimulation (HFS) of DBS. But the distinct results of both increase and decrease of spike firings of individual neurons create a paradox in explaining the effects of DBS (McIntyre et al., 2004a; Leblois et al., 2010; Cleary et al., 2013). Instead, rhythmic activity of local field potentials (LFP) could provide more relevant information of changes of neuronal networks associated with DBS (Kühn et al., 2005; Pogosyan et al., 2010; Lempka \& McIntyre, 2013). In addition, rhythmic events with excessive synchronization in neuronal networks have been reported to be responsible for many brain diseases (Uhlhaas \& Singer, 2006; Rossi et al., 2008; Thompson et al., 2014). Therefore, it is plausible that modulating the neuronal rhythms of LFP could be a mechanism underlying the efficacy of DBS (Karas et al., 2013; McIntyre et al., 2004b; Montgomery \& Baker, 2000).

However, few previous studies have directly shown the changes of LFP rhythms during HFS periods because of the interference of stimulation artifacts with high amplitudes. To avoid the artifacts, LFP signals collected after the termination of HFS have been used to infer the LFP activity during HFS (Barow et al., 2014; Wingeier et al., 2006; Foffani et al., 2006). Nevertheless, the neuronal activity under the stimulation of HFS could be different from the activity after the withdrawal of stimulation (Lehmkuhle et al., 2009; Swann et al., 2011). Thus, in the present study, we designed a proper algorithm to remove the artifacts in order to investigate the LFP changes induced by HFS of afferent axons in the hippocampal CA1 region of urethane-anesthetized rats. HFS-induced changes of LFP rhythms, i.e., $\delta, \theta, \alpha, \beta$ and $\gamma$ rhythms, were studied by power spectrum analysis and coherence analysis in different layers of the hippocampal CA1 region during 1-min HFS with a frequency of 100 or $200 \mathrm{~Hz}$.

LFP rhythms of hippocampal region (e.g., $\theta, \alpha$ and $\gamma$ rhythms) play important roles in physiological functions such as cognition, memory and emotions (Sirota et al., 2008; Klimesch, 1999; Basar et al., 1997; Fell et al., 2001; Sederberg et al., 2007; Gruber et al., 1999). Also, Hippocampus is a crucial structure for the brain functions of inhibition (Andersen et al., 2007). Thus, damages and abnormal activity in the hippocampal region could result in brain disorders such as epilepsy and AD that can potentially be treated by DBS (Hyman et al., 1984; Wasterlain et al., 1996; Bartsch \& Arzy, 2014; Laxton et al., 2012; Stam et al., 2002). Therefore, studying the modulation effects of DBS on the hippocampal LFP rhythms could provide new insights into advancing the clinical applications of DBS.

\section{Methods}

\subsection{Surgical procedures}

All procedures involving the animals and their care conformed to the Guide for the Care and Use of Laboratory Animals (China Ministry of Health). A total of 24 adult male Sprague Dawley rats (250-400g) were used in experiments. Each rat was 


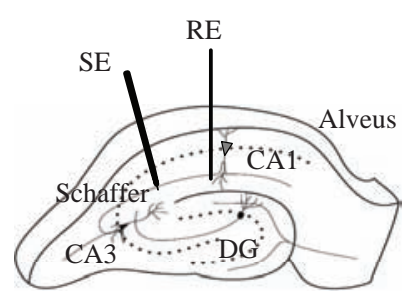

(a)

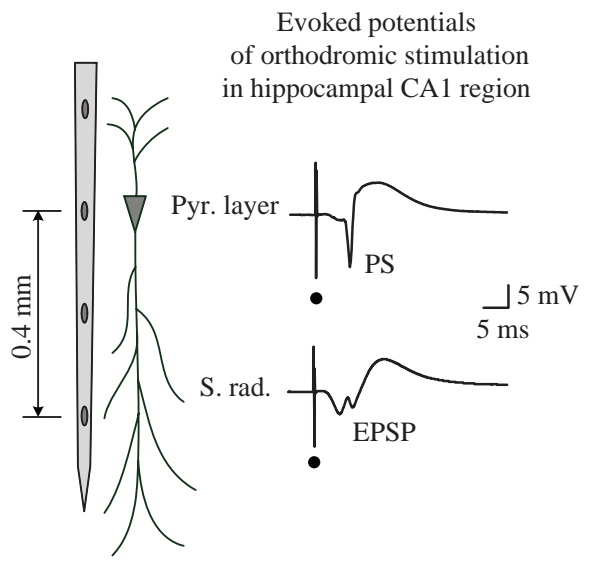

(b)

Fig. 1. Placement of electrodes and orthodromically evoked potentials of single pulse stimulation in the hippocampal CA1 region. (a) Schematic diagram of the locations of RE in the CA1 region and orthodromic SE in the Schaffer collaterals. (b) Left: A schematic drawing of CA1 pyramidal cell indicating the depths of the recording contacts. Right: the evoked potentials of single pulse stimulation recorded in the pyramidal layer (Pyr. layer) and in the stratum radiatum (S. rad.) by two contacts separated $0.4 \mathrm{~mm}$ vertically. Small dots below the potential waveforms denote the stimulation artifacts.

anesthetized with urethane $(1.25 \mathrm{~g} / \mathrm{kg}$, i.p.) and placed in a stereotaxic apparatus (Stoelting Co., Wood Dale, IL, USA). Part of the skull was removed. A microelectrode array (Model: A $1 \times 16$ or A $4 \times 4$, NeuroNexus Technologies, Ann Arbor, MI, USA,) was used as the recording electrode (RE) and was inserted into the hippocampal CA1 region (AP $3.5 \mathrm{~mm}$; ML $2.7 \mathrm{~mm}$; DV $2.3 \mathrm{~mm}$ ). A concentric bipolar metal electrode (Model CBBSC75, FHC, Bowdoin, ME 04287, USA) was inserted into the Schaffer collaterals of CA1 region (AP $2.2 \mathrm{~mm}$; ML $2.0 \mathrm{~mm}$; DV $2.8 \mathrm{~mm}$ ) and was used as the orthodromic stimulation electrode [SE, see Fig. 1(a)]. Two stainless steel screws were fixed into the nose bone and served as the reference electrode and the ground electrode, respectively. Patterns of population spike (PS) and excitatory post-synaptic potential (EPSP) evoked by orthodromic stimulation pulses [Fig. 1(b)] were used to determine the final positions of the recording array and the SE. Saline was put on the exposed surface of the dura to maintain moisture (Feng et al., 2013).

\subsection{Recording and stimulating}

Raw recording signals were amplified by a 16-channel extracellular amplifier (Model 3600, A-M system Inc., Carlsborg, WA, USA) with a frequency range of $0.3-5000 \mathrm{~Hz}$. The amplified signals were then sampled by a ML880 Powerlab 16/30 data acquisition system (ADInstruments Pty Ltd., NSW, Australia) at a sampling rate of $20 \mathrm{kHz} /$ channel with 16-bit precision, and were stored into a hard disk for offline analysis.

Biphasic-stimulation pulses with constant currents were generated by an isolated pulse stimulator (Model 2100, A-M system Inc., Carlsborg, WA, USA) with pulse 
duration of $0.1 \mathrm{~ms}$ per phase. An input-output (I/O) curve of orthodromic stimulation in the hippocampal CA1 region was obtained by applying stimulation pulses with increasing intensities from 0.1 to $0.8 \mathrm{~mA}$ with a $0.1 \mathrm{~mA}$ step for each experiment preparation. A current intensity $(0.3-0.5 \mathrm{~mA})$ that evoked PS with an amplitude approximately $3 / 4$ of the maximal PS amplitude in the I/O curves was used as the pulse intensity of HFS trains. The frequency of HFS was set as 100 or $200 \mathrm{~Hz}$, and the HFS duration was set as 1-min long. The intervals between sequential HFS trains were greater than 30 min to ensure recovery from previous stimulation (Feng et al., 2013).

\subsection{Removal of stimulation artifacts}

Stimulation artifacts of HFS in the raw recording signals contained abundant power components in a large frequency range. To avoid the influence of stimulation artifacts on the power spectrum analysis of LFP signals, we developed a custom-made MATLAB program (MathWorks, Inc., Natick, MA) incorporating an algorithm of linear interpolation to remove the artifacts. Since the slope of artifacts was much larger than that of neuronal electric signals, the absolute values of the first derivative of raw LFP sampling data were used to detect the stimulation artifacts automatically by a threshold method. Subsequently, a segment of 18 data points (i.e., $0.9 \mathrm{~ms}$ long sampling data) was replaced by interpolated data at each artifact location. The calculation of the $n$th point of interpolated data was:

$$
x_{n}=\frac{x_{N-1}-x_{0}}{\mathbf{N}} n+x_{0},
$$

where $x_{0}$ and $x_{N-1}$ were the values of first and last data of the interpolation segment, respectively. $N$ was the total number of data in each interpolated segment.

To verify this algorithm of artifact removal, a computer simulation was performed with a mimic LFP signal. The mimic pure LFP (termed as signal $\mathbf{R}$ ) was composed of sine waves of 5 different frequencies: $1,3,7,20$ and $45 \mathrm{~Hz}$, with amplitude ratio of 16:9:3:2:2. These sine waves represented 5 major rhythms of LFP appeared in anesthetized rats: $\delta(<2 \mathrm{~Hz}), \theta(2-5 \mathrm{~Hz}), \alpha(5-12 \mathrm{~Hz}), \beta(12-30 \mathrm{~Hz})$ and $\gamma(30-80 \mathrm{~Hz})$ (Buzsáki, 2002; Buzsaki, 2006; Kopell et al., 2000). Then, a train of stimulation artifacts of HFS was added to the signal $\mathbf{R}$ to form a 1-min long LFP signal with artifacts (termed as signal $\mathbf{P}$ ). The stimulation artifacts were extracted from real recordings with 100, 200 or $400 \mathrm{~Hz}$ HFS. The amplitude ratio of artifact to LFP was set as 40:1, according to real experiment recordings. Next, the artifacts within the signal $\mathbf{P}$ were removed by the linear interpolation algorithm to obtain an artifact-free signal (termed as signal $\mathbf{S}$ ). Finally, the power spectrum curves of the three signals $\mathbf{R}$, $\mathbf{P}$ and $\mathbf{S}$ were compared to evaluate the algorithm of artifact removal.

\subsection{Calculation of power spectrum and magnitude squared coherence for LFP signals}

To analyze the rhythms of LFP, we used the Labchart 7.3 software of the Powerlab data acquisition system to calculate the power spectrum density (PSD) and the 
time-frequency power spectrum of LFP signals in a frequency range of $0.3-80 \mathrm{~Hz}$. The PSD was calculated by Welch's modified periodogram method with a Hanning window of a width of $2^{17}=131,072$ sampling points $(\sim 6.4 \mathrm{~s}$ for the $20 \mathrm{kHz}$ sampling rate) and with a $50 \%$ overlap ratio. The frequency resolution of PSD was $0.153 \mathrm{~Hz}$. The time-frequency power spectra of LFP recordings were calculated by the method of short-time Fourier transform (Wang et al., 2005) with a Hanning window and an overlap ratio similar to the PSD calculation. The temporal resolution of time-frequency spectrum was $\sim 3.2 \mathrm{~s}$ and the frequency resolution was $0.153 \mathrm{~Hz}$.

To evaluate the linear synchronization between two LFP signals recorded in different sites, we used the index of magnitude squared coherence (MSC). The calculation formula of MSC for two recording signals $x$ and $y$ is:

$$
\operatorname{MSC}(f)=\frac{\left|\boldsymbol{S}_{x y}(f)\right|^{2}}{\boldsymbol{S}_{y y}(f) \boldsymbol{S}_{x x}(f)},
$$

where $S_{x y}(f)$ is the cross-power spectrum of the two signals, and $S_{y y}(f)$ and $S_{x x}(f)$ are the auto-power spectra of each individual signal. The MSC is a bounded measurement $(0 \leq \mathrm{MSC} \leq 1)$ (Zaveri et al., 1999). If the value of MSC of a certain frequency is closer to 1 , then the linear synchronization between the two signals at the frequency is higher; and vice versa.

The MSC was calculated by a custom-made MATLAB program using the build-in function of "mscohere" with a Hanning window of a width of $2^{17}$ sampling points and a $50 \%$ overlap ratio. The frequency resolution of MSC was also $0.153 \mathrm{~Hz}$, same as the PSD. Mean values of MSC were calculated in five frequency bands of $\delta, \theta, \alpha, \beta$ and $\gamma$ to evaluate the changes of synchronization in different LFP rhythms.

All data were represented statistically as mean \pm standard deviation. One-way ANOVA followed by post hoc Bonferroni tests were used to determine the statistical significance of the differences between multiple pairs of the three corresponding data obtained before, during and after HFS trains.

\section{Results}

\subsection{Verifying the algorithm of artifact removal}

Because the large artifacts of stimulation pulses during HFS trains would interfere with the analysis of LFP rhythms, a linear interpolation algorithm was used to remove the artifacts and to extract LFP signals submerged in the artifacts (Fig. 2).

To verify the algorithm of artifact removal, a computer simulation was performed as shown in Fig. 3(a). The mimic LFP signal (signal $\mathbf{P}$ ) was composed of pure LFP (signal $\mathbf{R}$, composed of 5 sine waves with frequencies of 1, 3, 7, 20 and $45 \mathrm{~Hz}$ ) as well as stimulation artifacts. Signal $\mathbf{S}$ was obtained after removing the artifacts in the signal $\mathbf{P}$. The plots of PSD show [Fig. 3(b)] that the PSD curve of signal $\mathbf{S}$ was almost superimposed with the PSD curve of pure LFP, indicating validity of the artifact removal. The power ratios of $|\mathbf{S}-\mathbf{R}| / \mathbf{R}$ in the five frequency bands $(\delta, \theta, \alpha, \beta$ and $\gamma)$ 


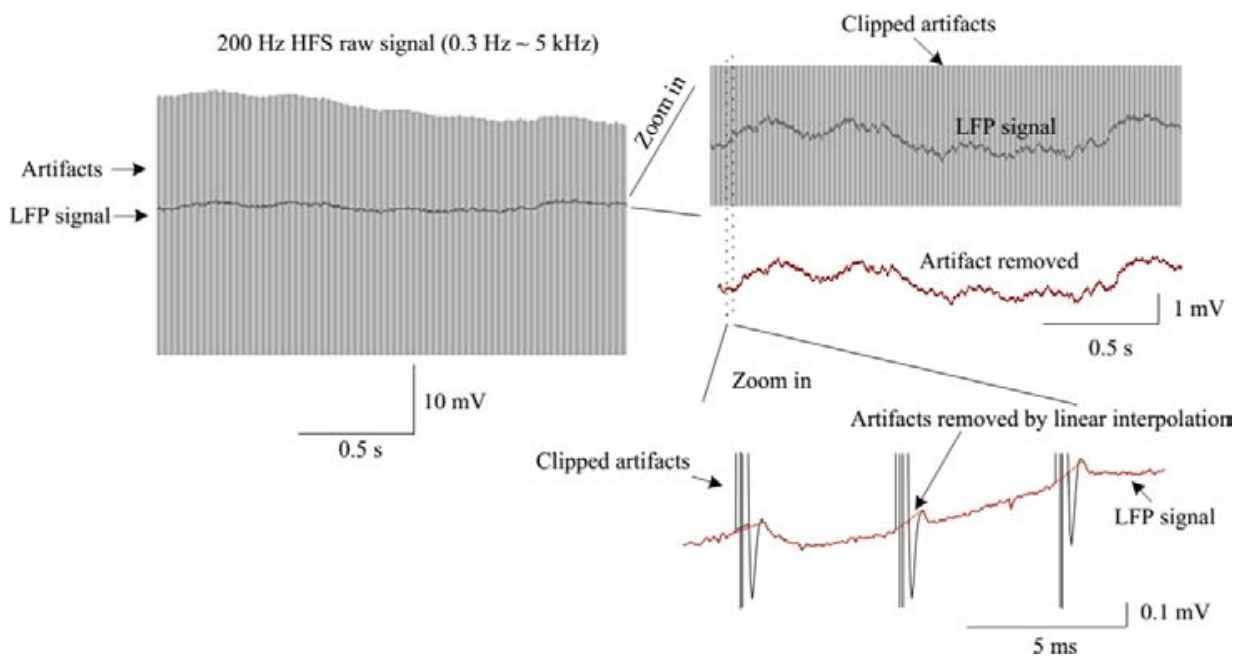

Fig. 2. Extracting LFP signals submerged in the stimulation artifacts during HFS trains. A typical segment (left) of raw recording signals during HFS period and the schematic diagram (right) of artifact removal algorithm of linear interpolation. The artifacts were identified by setting a slope threshold and were replaced by interpolated data. The expanded inset at the right bottom shows the overlapped waveforms of the raw signal and the artifact-free LFP signal after artifact removal.

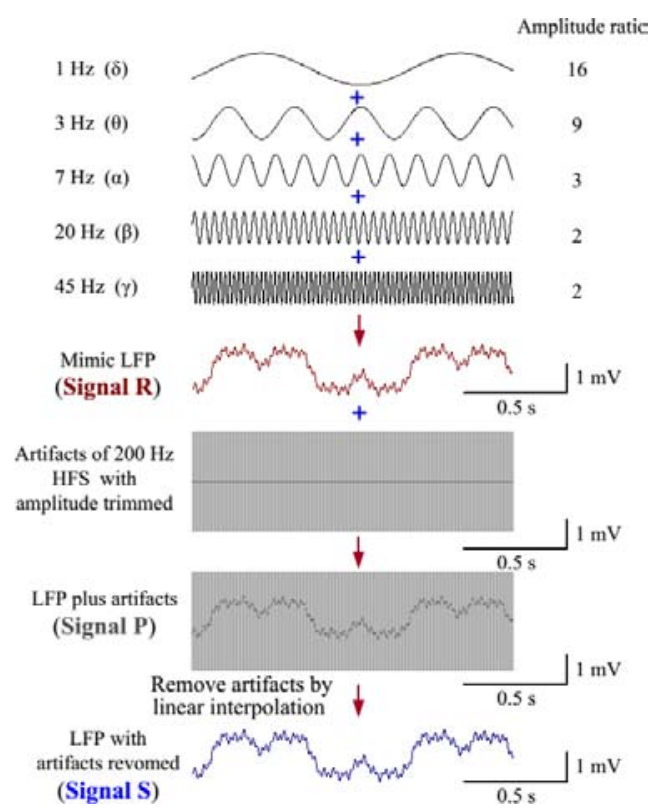

(a)

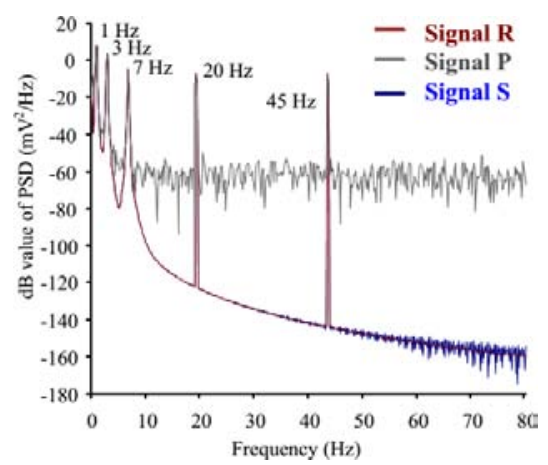

(b)

Fig. 3. Verifying the method of artifact removal by a computer simulation. (a) Construction of mimic LFP signals with stimulation artifacts and artifact removal by an algorithm of linear interpolation. The mimic LFP (signal R) was composed of sine waves of 5 frequencies of $1,3,7,20$ and $45 \mathrm{~Hz}$ with amplitude ratios of 16:9:3:2:2. The LFP with artifacts (signal $\mathbf{P}$ ) was mimicked by adding the stimulation artifacts of HFS to the signal R. A linear interpolation algorithm was applied to remove the artifacts within signal $\mathbf{P}$ to obtain an artifact-free signal (signal $\mathbf{S}$ ). (b) Comparison of the PSD curves of signal $\mathbf{R}$, signal $\mathbf{P}$ and signal $\mathbf{S}$. 
were calculated to evaluate the distortion generated by the interpolation algorithm of artifact removal. The maximum values of $|\mathbf{S}-\mathbf{R}| / \mathbf{R}$ out of the 5 rhythms were $0.13 \%, 0.26 \%$ and $0.51 \%$ for 100,200 and $400 \mathrm{~Hz}$ HFS, respectively.

These results indicate that the linear interpolation algorithm did not distort the LFP rhythms markedly even when the frequency of HFS was as high as $400 \mathrm{~Hz}$. Therefore, LFP signals obtained after artifact removal could be used to investigate the effects of electrical stimulations on the LFP rhythms that reflect the activity of neural networks even during HFS periods.

\subsection{Modulation effects of axonal HFS on LFP rhythms in the hippocampal $C A 1$ region}

To investigate the effects of axonal HFS on the rhythms of LFP in the downstream CA1 region, we applied 1-min long HFS at the Schaffer collaterals of CA1 region. Upon application of $200 \mathrm{~Hz}$ HFS, large PS and EPSP were evoked instantaneously by the stimulation [Fig. 4(a)]. However, after the initial tens of seconds of HFS, these evoked potentials disappeared rapidly and did not reappear for the remaining period of HFS. Though no more PS events appeared during the late period of HFS, the multiple unit activity (MUA) increased with the appearance of unit spikes that were absent in the baseline recording before HFS [see the expanded waveforms at the bottom of Fig. 4(a)]. These results indicate that the HFS pulses still had excitatory effect on the neurons at downstream area but with a decreased strength not strong enough to evoke large PS events.

In addition, the $\theta$ rhythm $(\sim 3.5 \mathrm{~Hz})$ in the baseline recording (especially in the stratum radiatum of CA1) decreased markedly during HFS and recovered after HFS [see the expanded waveforms in Fig. 4(a)]. HFS-induced changes of LFP rhythms were further revealed in time-frequency power spectra [Fig. 4(b)]. In the pyramidal layer, the power of LFP in the frequency range of $30-80 \mathrm{~Hz}$ (i.e., $\gamma$ rhythm) increased. In the stratum radiatum, the power of LFP in the range of $2-30 \mathrm{~Hz}$ (i.e., $\theta, \alpha$ and $\beta$ rhythms) decreased. Especially, the $\theta$ rhythm, represented by the bright yellow strip in the spectrum of stratum radiatum before HFS, disappeared during HFS and reappeared after HFS. This result indicates that HFS could suppress $\theta$ rhythm in the stratum radiatum of $\mathrm{CA} 1$ region.

To avoid the interference from evoked population potentials, the late $35 \mathrm{~s}$ period of 1-min long HFS was used to calculate PSD of LFP induced by HFS. LFP powers were calculated for the five frequency bands of $\delta, \theta, \alpha, \beta$ and $\gamma$ recorded both in the pyramidal layer and in the stratum radiatum of CA1 region before HFS (1-min long), during HFS (35 s long) and after HFS (1-min long), as shown in Fig. 5. In addition, two stimulation frequencies of $100 \mathrm{~Hz}$ [Fig. 5(a)] and $200 \mathrm{~Hz}$ [Fig. 5(b)] were investigated since the effective frequency of DBS has been shown in the range of 100$200 \mathrm{~Hz}$ (Wingeier et al., 2006).

During both 100 and $200 \mathrm{~Hz}$ HFS, in the pyramidal layer, the $\gamma$ power of LFP increased significantly, while the powers of other rhythms showed no significant 


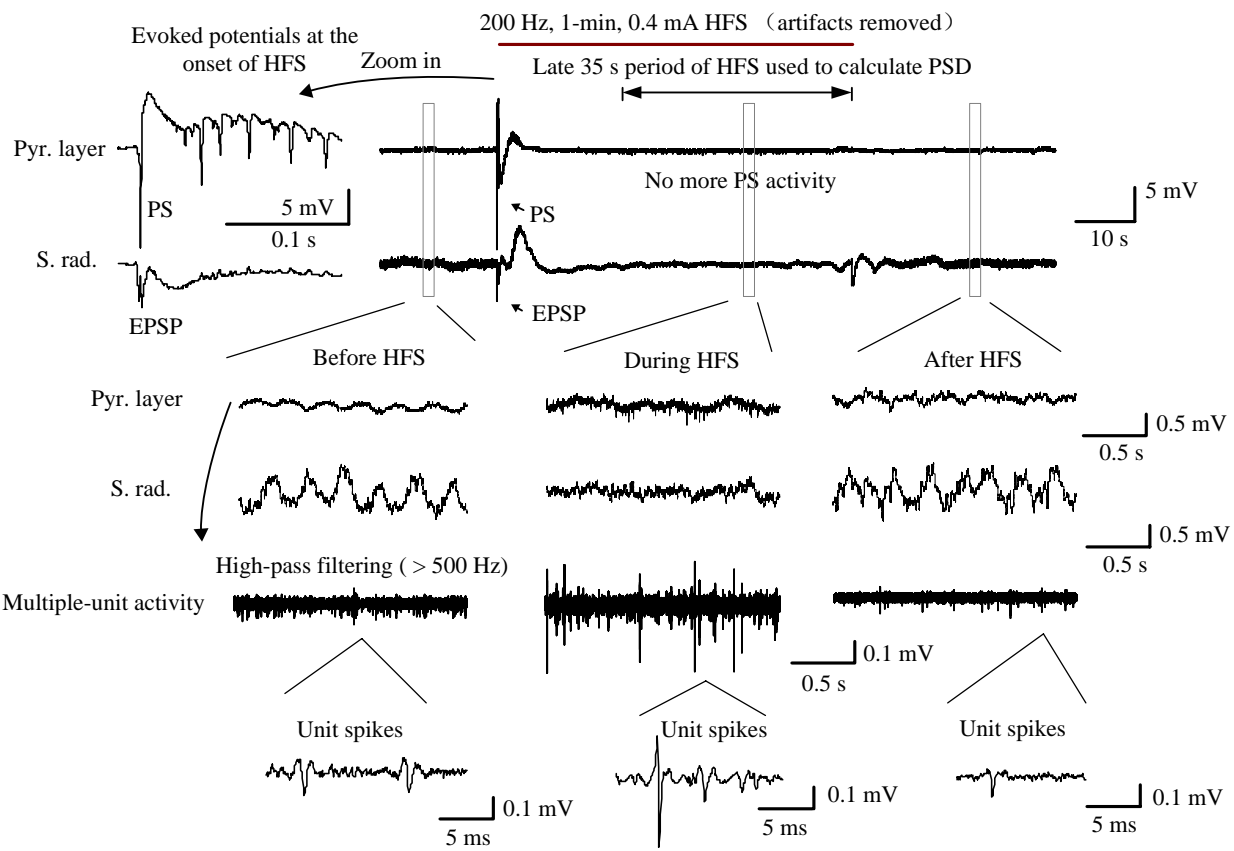

(a)

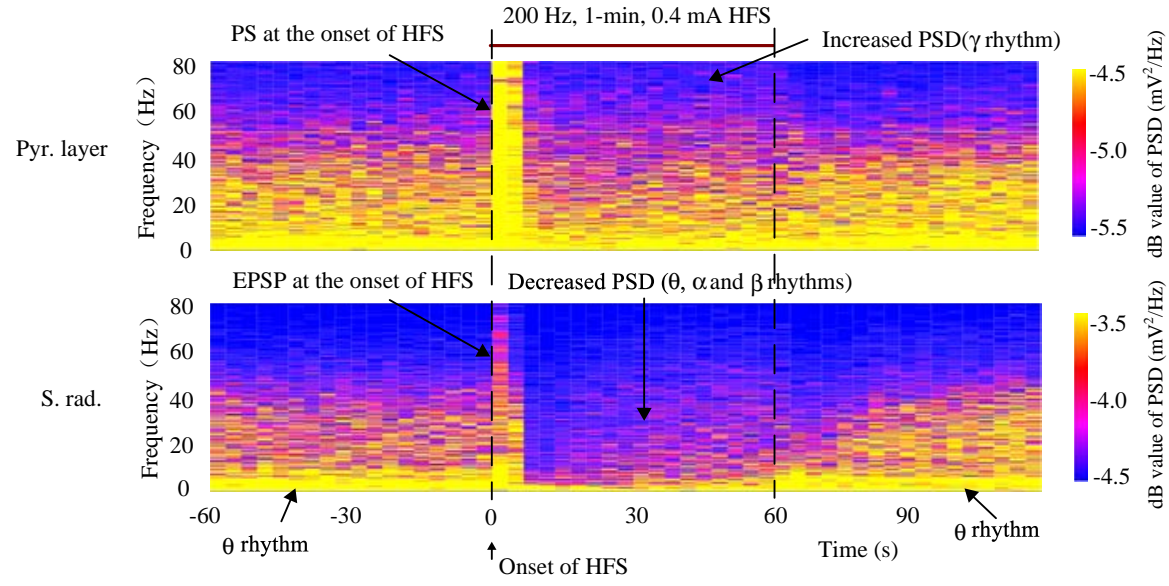

(b)

Fig. 4. (Color online) Changes of LFP induced by HFS of afferent axons in the hippocampal CA1 region. (a) Typical signals recorded in the Pyr. layer and the S. rad. of CA1 region with a 1-min long $200 \mathrm{~Hz}$ HFS train. The red bar above the signals denotes the duration of HFS. At the beginning of HFS, large PS and EPSP were evoked by the stimulation pulses in the Pyr. layer and the S. rad. (the expanded insets on the top left). These evoked potentials disappeared rapidly and did not reappear in the late period of HFS. However, obvious changes of LFP rhythms persisted and the MUA still increased during the late period of HFS as compared to baseline MUA before HFS (the expanded insets on the bottom). The MUA signal was extracted by high-pass filtering the artifact-free signals recorded in the pyramidal layer. (b) The time-frequency power spectra of the LFP signals. During HFS, the power of LFP in the frequency range of $30-80 \mathrm{~Hz}$ (i.e., $\gamma$ rhythm) increased in the Pyr. layer, while the power of LFP in the range of $2-30 \mathrm{~Hz}$ (i.e., $\theta, \alpha$ and $\beta$ rhythms) decreased in the S. rad. The $\theta$ rhythm in the S. rad., represented by the bright yellow strip, disappeared during HFS. 


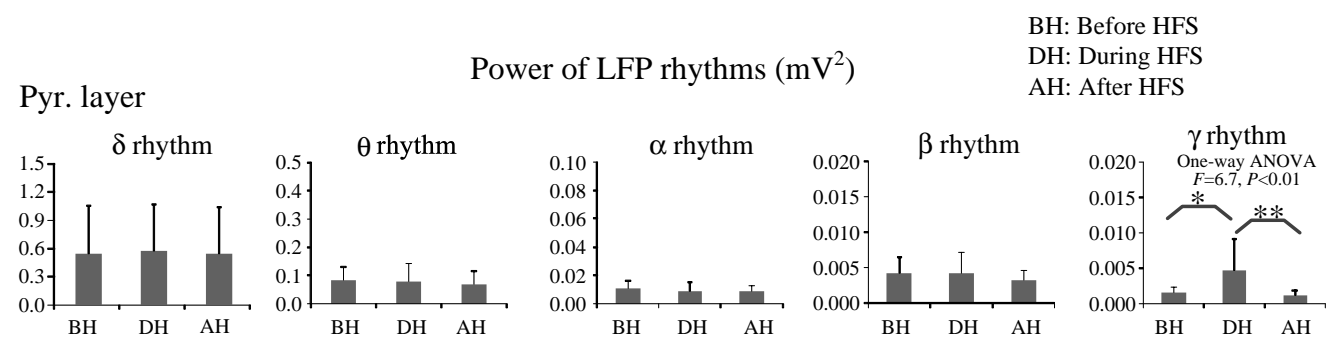

S. rad.
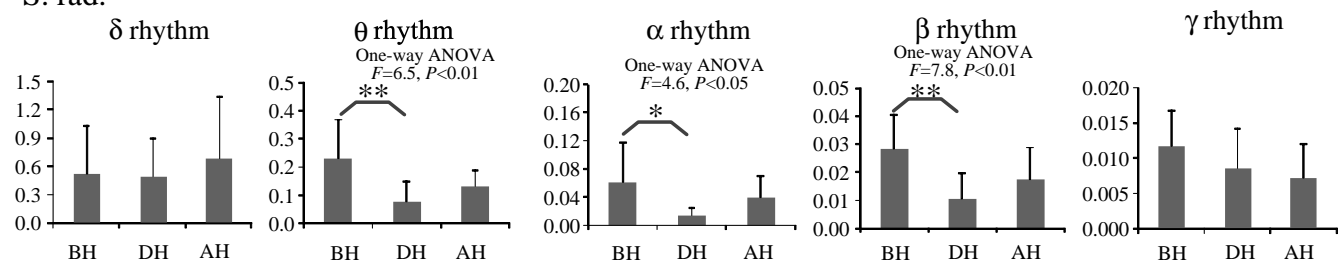

(a) $100 \mathrm{~Hz} \operatorname{HFS}(n=13)$
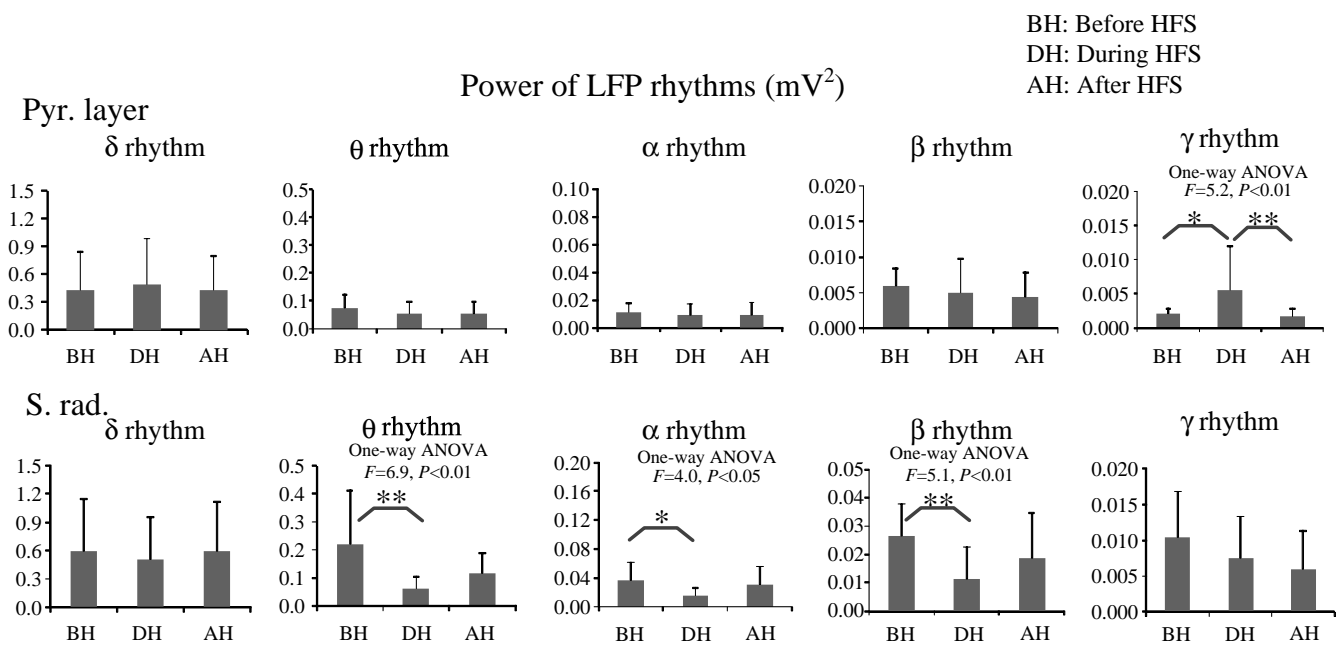

(b) $200 \mathrm{~Hz}$ HFS $(n=16)$

Fig. 5. Changes of mean power in the Pyr. layer and the S. rad. in the 5 frequency bands of $\delta(0.3-$ $2 \mathrm{~Hz}), \theta(2-5 \mathrm{~Hz}), \alpha(5-12 \mathrm{~Hz}), \beta(12-30 \mathrm{~Hz})$ and $\gamma(30-80 \mathrm{~Hz})$ before, during and after $100 \mathrm{~Hz} \mathrm{HFS}$ (a, $n=13 \mathrm{rats}$ ) and $200 \mathrm{~Hz}$ HFS (b, $n=16 \mathrm{rats}) .{ }^{*} p<0.05$ and ${ }^{* *} p<0.01$, post hoc Bonferroni test.

change. In the stratum radiatum, the power in the frequency range of $2-30 \mathrm{~Hz}$ (i.e., $\theta$, $\alpha$ and $\beta$ rhythms) decreased significantly, while the $\gamma$ power had no significant change. After HFS, the LFP powers recovered to the baseline level before HFS (Fig. 5).

Taken together, during the late period of HFS without evoked population potentials, the HFS of Schaffer collaterals increased the LFP rhythms of higher frequency in the pyramidal layer and decreased the LFP rhythms of lower frequency 
in the apical dendrite layer of CA1 region. These results indicate that the HFS of afferent axon could modulate the neuronal activity in the downstream area.

\subsection{Modulation effects of axonal HFS on the synchronization of LFP signals from different neuronal populations}

LFP is considered as the integrated potentials of a neuronal population located near the electrode contact, including synaptic potentials in a radius about $0.35 \mathrm{~mm}$ (Vianney-Rodrigues et al., 2011). To investigate the effects of HFS on the synchronization of LFP rhythms from different neuronal populations, we next analyzed the changes of coherence between pairs of LFP signals recorded from two different electrode contacts. The MSC was calculated for each of the two pairs of LFP signals recorded by contact pairs located either in the pyramidal layer or in the stratum radiatum of CA1 region [Fig. 6(a)]. The two recording contacts for each pair were separated horizontally by $0.6 \mathrm{~mm}$ distance.

Example segments of LFP signals are shown in pairs in Fig. 6(a). Strongly synchronized $\theta$ rhythms were exhibited between the LFP pairs before HFS and after HFS, both in the pyramidal layer and in the stratum radiatum. However, during HFS, in the stratum radiatum, with a power decrease of $\theta$ rhythms, the synchronization of the LFP pair also decreased.

Mean MSC values of five LFP rhythms $(\delta, \theta, \alpha, \beta$ and $\gamma)$ both in the pyramidal layer and in the stratum radiatum of CA1 region were statistically compared before, during and after 100 and $200 \mathrm{~Hz}$ HFS [Fig. 6(b)]. During both 100 and $200 \mathrm{~Hz}$ HFS, the MSC values of all LFP rhythms in the pyramidal layer did not change significantly compared with before HFS. However, the MSC values of $\theta$ rhythm in the stratum radiatum decreased significantly during 100 and $200 \mathrm{~Hz} \mathrm{HFS}$, and the MSC values of $\gamma$ rhythm increased significantly during $200 \mathrm{~Hz}$ HFS, compared with before HFS.

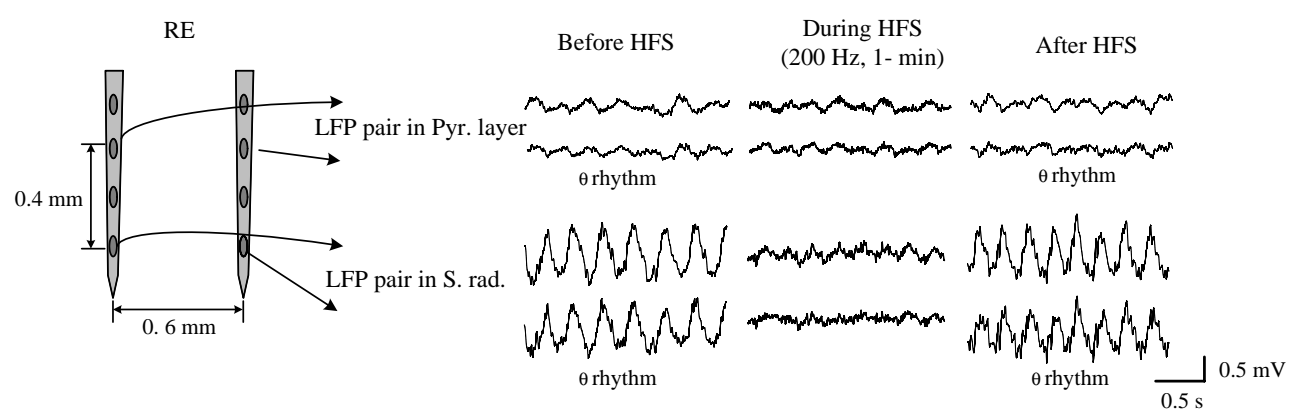

(a)

Fig. 6. HFS-induced changes of the synchronization of LFP rhythms in two different layers of neuronal populations. (a) Left: Schematic diagram of the two pairs of LFP recordings in two depths (Pyr. layer and S. rad.) in the CA1 region. Right: Short segments of signal examples extracted before, during and after HFS show the changes of LFP rhythms and the synchronization relationship between each LFP pair. (b) Mean MSC of the two LFP pairs in the Pyr. layer (top) and in the S. rad. (bottom) in the 5 frequency bands of $\delta, \theta, \alpha, \beta$ and $\gamma$ induced by 100 and $200 \mathrm{~Hz}$ HFS $\left(n=8\right.$ rats). ${ }^{*} p<0.05$, Post hoc Bonferroni test with one-way ANOVA $F>3.6, p<0.05$. 

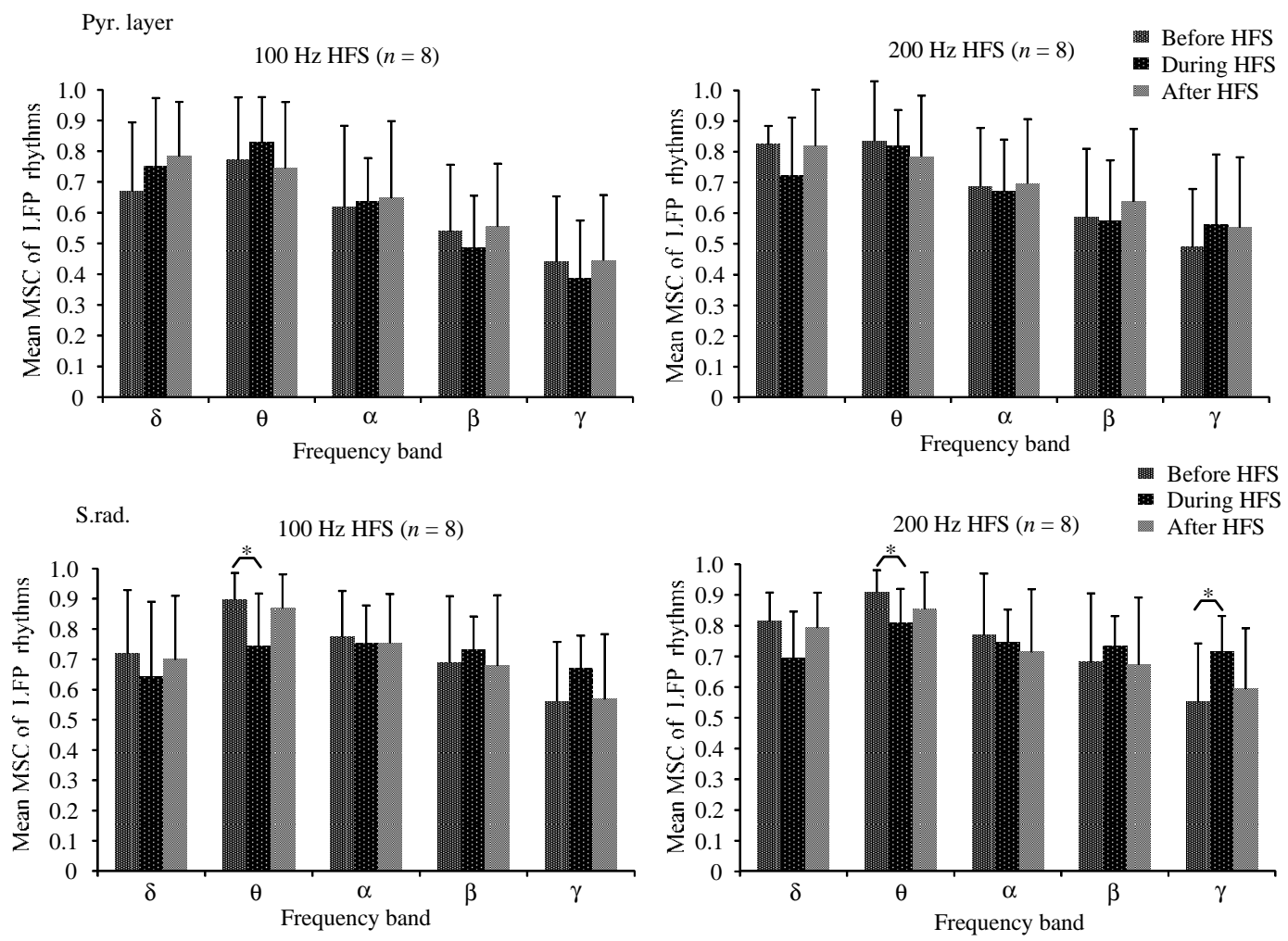

(b)

Fig. 6. (Continued)

In addition, the mean MSC values of LFP in the lower frequency ranges (e.g., $\delta$ and $\theta$ rhythm) were larger than those in the higher frequency ranges (e.g., $\gamma$ rhythm) both in the pyramidal layer and in the stratum radiatum [Fig. 6(b)], indicating a lowpass effect of the brain tissue that attenuated the propagation of high-frequency components of LFP.

These results indicate that HFS of CA1 afferent axons would not increase the synchronization of LFP in the pyramidal layer in the higher frequency range of $\gamma$ rhythm, even though the $\gamma$ power increased during HFS (see Fig. 5). Nevertheless, the synchronization of LFP in the stratum radiatum decreased in the lower frequency range of $\theta$ rhythm with the decrease of $\theta$ power (see Fig. 5). The results indicate that HFS modulated the activity of different neuronal populations in downstream areas independently.

\section{Discussion}

In the present study, the changes of LFP rhythms in the hippocampal CA1 region by the HFS of afferent axons were investigated after artifact removal. The major findings include: (1) the method of artifact removal with a linear interpolation algorithm was valid to remove the HFS artifacts without distorting LFP rhythms. (2) The 
power of higher frequency rhythms in the pyramidal layer increased and the power of lower frequency rhythms in the stratum radiatum decreased during HFS. (3) The synchronization of $\theta$ rhythm decreased and the synchronization of $\gamma$ rhythm increased during HFS between two LFP signals recorded in two different sites in the stratum radiatum of $\mathrm{CA} 1$.

\subsection{Artifact removal for LFP analysis during HFS}

During HFS, small LFP signals were submerged in the large and dense stimulation artifacts. Therefore, many previous studies investigated the LFP signals after the termination of HFS to infer the LFP activity during HFS (Barow et al., 2014; Wingeier et al., 2006; Foffani et al., 2006). However, the LFP activity could recover rapidly after HFS (Fig. 4). In the present study, we applied a linear interpolation algorithm to remove the artifacts. The process of artifact removal was applied to a mimic LFP with artifacts and frequency components representative of five frequency bands, and was verified by a computer simulation. Because the individual removal segment of an artifact was shorter than $1 \mathrm{~ms}$ and only a fraction of the period $(>12.5 \mathrm{~ms})$ of LFP rhythms $(<80 \mathrm{~Hz})$, the process of artifact removal did not distort the inherent LFP rhythms. The HFS-induced changes of LFP rhythms in the hippocampal CA1 region were clearly revealed after the artifact removal.

\subsection{Plausible underlying mechanisms for the HFS-induced changes of LFP rhythms}

The neurons of hippocampal region are arranged tightly in a clear hierarchy. Therefore, a single pulse of stimulation at the Schaffer collaterals of the CA1 afferent pathway with intensity above threshold would induce large PS and EPSP in the downstream CA1 region [Fig. 1(b)]. However, trains of persistent pulses with high frequency of $100-200 \mathrm{~Hz}$ could induce a conduction blockage at axonal fibers that would prevent the pulses to evoke larger population potentials as shown in previous studies (Feng et al., 2013, 2014) and in the present study [Fig. 4(a)]. Presumably, this HFS-induced conduction blockage would cause changes in LFP rhythms of the downstream region, particularly the $\theta$ rhythm (Fig. 4).

The $\theta$ rhythm of LFP in the hippocampal CA1 region often appear during waking state of freely moving rats or appear in urethane-anesthetized rats. As shown in the recordings of the present study (Fig. 4), the $\theta$ frequency in anesthetized rats $(2-5 \mathrm{~Hz})$ is lower than that in awake rats $(6-9 \mathrm{~Hz})$ (Buzsáki, 2002). The generation of the $\theta$ rhythm of CA1 is associated with the excitatory inputs from CA3 via the Schaffer collaterals. The CA3 region functions as an intra-hippocampal $\theta$ oscillator which is responsible for the emergence and control of the oscillatory pattern and frequency in the hippocampus (Buzsáki, 2002). HFS-induced axonal blockage of the Schaffer collaterals might disconnect the CA1 region from the sources of $\theta$ rhythm in the CA3 region. This may explain the present finding that the $\theta$ power of LFP decreased during HFS. The decrease was significant in the stratum radiatum of CA1 (Fig. 5) 
because large $\theta$ rhythm appears in this layer where Schaffer collaterals form synapses with the apical dendrites of the pyramidal cells of CA1 region (Kamondi et al., 1998; Buzsáki, 2002). Presumably, the decrease of synchronization of LFP $\theta$ rhythm between different neuronal populations in the stratum radiatum of CA1 might also be caused by the lack of the rhythmical controls from upstream region of CA3.

Other lower frequency rhythms of LFP (e.g., $\alpha$ and $\beta$ rhythms) in the hippocampus have been considered to originate from cortex or sub-thalamic nucleus outside of hippocampus (Bollimunta et al., 2008; Buzsáki, 2006; Avila et al., 2010). The block of excitatory inputs of CA1 by HFS could affect the information conducting from external regions to the CA1 region, thereby decreasing these lower frequency rhythms of LFP in the stratum radiatum of CA1 region.

The increase of higher frequency $\gamma$ power of LFP in the CA1 pyramidal layer may be caused by the increase of MUA [Fig. 4(a)]. This is consistent with previous studies suggesting that an increase of action potential firings of single neurons can increase the $\gamma$ power of LFP (Ray \& Maunsell, 2011). The increase of MUA during HFS could be attributed to attenuated actions of the stimulation pulses resulting from a partial blockage of the Schaffer collaterals by HFS (Jensen \& Durand, 2009; Feng et al., 2013). The attenuated actions of HFS could activate scattered firings of individual pyramidal cells but might not be strong enough to activate a large population of the cells simultaneously. The waveforms of unit spikes are composed of high-frequency components that have a limited propagation distance in space. Therefore, the synchronization of $\gamma$ rhythm in the pyramidal layer did not increase with the increase of $\gamma$ power. The increase of the synchronization of $\gamma$ rhythm in the stratum radiatum might be caused by the direct action of the stimulation pulses of HFS nearby (Tass et al., 2009).

In addition, a short train of HFS (e.g., $1 \mathrm{~s}$ and $100 \mathrm{~Hz}$ pulses) in the Schaffer collaterals can induce long-term potentiation (LTP) in the synapses of apical dendrites of hippocampal CA1 region indicated by an increase of post-synaptic evokedpotentials (Bliss \& Lomo, 1973; Bliss \& Collingridge, 1993). However, no increases of evoked potentials have been observed after minute long trains of HFS (Feng et al., 2013), indicating that LTP might not be the mechanism of long HFS.

Taken together, the HFS of afferent axons may modulate LFP rhythms in the downstream region by decreasing the lower frequency components and increasing the higher frequency components.

In fact, previous studies have suggested that modulation of rhythmic activity in neural network could be an underlying mechanism of DBS. For instance, sine-wave stimulation of ventral hippocampus of rats could induce reduction of LFP $\theta$ rhythm in CA1 region (La Corte et al., 2014). DBS of sub-thalamic nucleus in patients and rats who suffered from Parkinson disease could decrease abnormal rhythms of low frequency $(<30 \mathrm{~Hz})$ in LFP and in electrocorticogram (Eusebio et al., 2011; Lehmkuhle et al., 2009). In agreement with these reports, our results of the present study provided novel evidence of LFP modulation in the hippocampal CA1 region after removing stimulation artifacts. Moreover, we suggested a mechanism of HFS-induced 
conduction blockage of afferent axons to explain the modulation of LFP rhythms by DBS. To the best of our knowledge, this mechanism has not been proposed previously.

\subsection{Conclusion}

In conclusion, the present study showed that axonal HFS could modulate LFP rhythms in the downstream brain areas in hippocampal region with a plausible underlying mechanism of HFS-induced axonal blockage. Literature has suggested that patients with $\mathrm{AD}$, a final stage of amnesic mild cognitive impairment (Deiber et al., 2008; Petersen et al., 2001), present higher $\theta$ rhythm activity (Başar \& Güntekin, 2013) and lower $\gamma$ rhythm activity (Stam et al., 2002) in electroencephalograms than healthy persons. The inverse modulation of $\theta$ and $\gamma$ rhythms in the hippocampal LFP in the present study indicates a potential therapy of HFS to AD patients. Therefore, the novel finding of LFP modulation by HFS of afferent axons may shed lights on advancing the clinical application of DBS.

\section{Acknowledgments}

This work was supported by the National Natural Science Foundation of China (No. 30970753) and by Major State Basic Research Development Program of China (No. 2011CB504400).

\section{REFERENCES}

Andersen, P., Morris, R., Amaral, D., Bliss, T. \& O'Keefe, J., eds. (2007) The Hippocampus Book. Oxford, England: Oxford University Press, p. 582.

Avila, I., Parr-Brownlie, L.C., Brazhnik, E., Castañeda, E., Bergstrom, D.A. \& Walters, J.R. (2010) Beta frequency synchronization in basal ganglia output during rest and walk in a hemiparkinsonian rat. Exp. Neurol., 221, 307-319.

Barow, E., Neumann, W.J., Brücke, C., Huebl, J., Horn, A., Brown, P., Krauss, J.K., Schneider, G. \& Kühn, A.A. (2014) Deep brain stimulation suppresses pallidal low frequency activity in patients with phasic dystonic movements. Brain, 137, 3012-3024.

Bartsch, T. \& Arzy, S. (2014) Human memory: Insights into hippocampal networks in epilepsy. Brain, 137, 1856-1857.

Başar, E. \& Güntekin, B. (2013) Review of delta, theta, alpha, beta, and gamma response oscillations in neuropsychiatric disorders. Suppl. Clin. Neurophysiol., 62, 303-341.

Basar, E., Schurmann, M., Basar-Eroglu, C. \& Karakas, S. (1997) Alpha oscillations in brain functioning: An integrative theory. Int. J. Psychophysiol., 26, 5-29.

Bliss, T.V. \& Collingridge, G.L. (1993) A synaptic model of memory: Longterm potentiation in the hippocampus. Nature, 361, 31-39.

Bliss, T.V. \& Lomo, T. (1973) Long-lasting potentiation of synaptic transmission in the dentate area of the anaesthetized rabbit following stimulation of the perforant path. J. Physiol., 232, 331-356.

Bollimunta, A., Chen, Y., Schroeder, C.E. \& Ding, M. (2008) Neuronal mechanisms of cortical alpha oscillations in awake-behaving macaques. J. Neurosci., 28, 9976-9988. 
Boon, P., Vonck, K., De Herdt, V., Van Dycke, A., Goethals, M., Goossens, L. \& Van Roost, D. (2007) Deep brain stimulation in patients with refractory temporal lobe epilepsy. Epilepsia, 48, 1551-1560.

Buzsáki, G. (2002) Theta oscillations in the hippocampus. Neuron, 33, 325-340.

Buzsaki, G. (2006) Rhythms of the Brain. Oxford, England: Oxford University Press, pp. $195-196$.

Cleary, D.R., Raslan, A.M., Rubin, J.E., Bahgat, D., Viswanathan, A., Heinricher, M. M. \& Burchiel, K.J. (2013) Deep brain stimulation entrains local neuronal firing in human globus pallidus internus. J. Neurophysiol., 109, 978-987.

Deiber, M.P., Ibanez, V., Missonnier, P., Herrmann, F., Fazio-Costa, L., Gold, G. \& Giannakopoulos, P. (2008) Abnormal-induced theta activity supports early directedattention network deficits in progressive MCI. Neurobiol. Aging, 30, 1444-1452.

Eusebio, A., Thevathasan, W., Gaynor, L.D., Pogosyan, A., Bye, E., Foltynie, T., Zrinzo, L., Ashkan, K. \& Brown, P. (2011) Deep brain stimulation can suppress pathological synchronisation in parkinsonian patients. J. Neurol. Neurosurg. Psychiatry, 82, 569-573.

Fell, J., Klaver, P., Lehnertz, K., Grunwald, T., Schaller, C., Elger, C.E. \& Fernández, G. (2001) Human memory formation is accompanied by rhinal-hippocampal coupling and decoupling. Nat. Neurosci., 4, 1259-1264.

Feng, Z., Yu, Y., Guo, Z., Cao, J. \& Durand, D.M. (2014) High frequency stimulation extends the refractory period and generates axonal block in the rat hippocampus. Brain Stimul., $\mathbf{7}$, $680-689$.

Feng, Z., Zheng, X., Yu, Y. \& Durand, D.M. (2013) Functional disconnection of axonal fibers generated by high frequency stimulation in the hippocampal CA1 region in vivo. Brain Res., 1509, 32-42.

Foffani, G., Ardolino, G., Egidi, M., Caputo, E., Bossi, B. \& Priori, A. (2006) Subthalamic oscillatory activities at beta or higher frequency do not change after high-frequency DBS in Parkinson's disease. Brain Res. Bull., 69, 123-130.

Gruber, T., Müller, M.M., Keil, A. \& Elbert, T. (1999) Selective visual-spatial attention alters induced gamma band responses in the human EEG. Clin. Neurophysiol., 110, 2074-2085.

Hescham, S., Lim, L.W., Jahanshahi, A., Steinbusch, H.W., Prickaerts, J., Blokland, A. \& Temel, Y. (2013) Deep brain stimulation of the forniceal area enhances memory functions in experimental dementia: The role of stimulation parameters. Brain Stimul., 6, 72-77.

Hyman, B.T., Van Hoesen, G.W., Damasio, A.R. \& Barnes, C.L. (1984) Alzheimer's disease: Cell-specific pathology isolates the hippocampal formation. Science, 225, 1168-1170.

Jensen, A.L. \& Durand, D.M. (2009) High frequency stimulation can block axonal conduction. Exp. Neurol., 220, 57-70.

Kamondi, A., Acsády, L., Wang, X.J. \& Buzsáki, G. (1998) Theta oscillations in somata and dendrites of hippocampal pyramidal cells in vivo: Activity-dependent phase-precession of action potentials. Hippocampus, 8, 244-261.

Karas, P.J., Mikell, C.B., Christian, E., Liker, M.A. \& Sheth, S.A. (2013) Deep brain stimulation: A mechanistic and clinical update. Neurosurg. Focus, 35, E1.

Klimesch, W. (1999) EEG alpha and theta oscillations reflect cognitive and memory performance: A review and analysis. Brain Res. Rev., 29, 169-195.

Kopell, N., Ermentrout, G.B., Whittington, M.A. \& Traub, R.D. (2000) Gamma rhythms and beta rhythms have different synchronization properties. Proc. Natl. Acad. Sci. USA, 97, 1867-1872. 
Kühn, A.A., Trottenberg, T., Kivi, A., Kupsch, A., Schneider, G.H. \& Brown, P. (2005) The relationship between local field potential and neuronal discharge in the subthalamic nucleus of patients with Parkinson's disease. Exp. Neurol., 194, 212-220.

La Corte, G., Wei, Y., Chernyy, N., Gluckman, B.J. \& Schiff, S.J. (2014) Frequency dependence of behavioral modulation by hippocampal electrical stimulation. J. Neurophysiol., 111, 470480 .

Laxton, A.W., Sankar, T., Lozano, A.M. \& Hamani, C. (2012) Deep brain stimulation effects on memory. J. Neurosurg. Sci., 56, 341-344.

Leblois, A., Reese, R., Labarre, D., Hamann, M., Richter, A., Boraud, T. \& Meissner, W.G. (2010) Deep brain stimulation changes basal ganglia output nuclei firing pattern in the dystonic hamster. Neurobiol. Dis., 38, 288-298.

Lehmkuhle, M.J., Bhangoo, S.S. \& Kipke, D.R. (2009) The electrocorticogram signal can be modulated with deep brain stimulation of the subthalamic nucleus in the hemiparkinsonian rat. J. Neurophysiol., 102, 1811-1820.

Lempka, S.F. \& McIntyre, C.C. (2013) Theoretical analysis of the local field potential in deep brain stimulation applications. PloS One, 8, e59839.

McGovern, R.A., Sheehy, J.P., Zacharia, B.E., Chan, A.K., Ford, B. \& McKhann, G.M. (2013) Unchanged safety outcomes in deep brain stimulation surgery for Parkinson disease despite a decentralization of care: Clinical article. J. Neurosurg., 119, 1546-1555.

McIntyre, C.C., Grill, W.M., Sherman, D.L. \& Thakor, N.V. (2004a) Cellular effects of deep brain stimulation: Model-based analysis of activation and inhibition. J. Neurophysiol., 91, 1457-1469.

McIntyre, C.C., Savasta, M., Kerkerian-Le Goff, L. \& Vitek, J.L. (2004b) Uncovering the mechanism (s) of action of deep brain stimulation: Activation, inhibition, or both. Clin. Neurophysiol., 115, 1239-1248.

Montgomery Jr., E.B. \& Baker, K.B. (2000) Mechanisms of deep brain stimulation and future technical developments. Neurol. Res., 22, 259-266.

Petersen, R.C., Doody, R., Kurz, A., Mohs, R.C., Morris, J.C., Rabins, P.V. Ritchie, K., Rossor, M., Thal, L. \& Winblad, B. (2001) Current concepts in mild cognitive impairment. Arch. Neurol., 58, 1985-1992.

Pogosyan, A., Yoshida, F., Chen, C.C., Martinez-Torres, I., Foltynie, T., Limousin, P., Zrinzo, L., Hariz, M.I. \& Brown, P. (2010) Parkinsonian impairment correlates with spatially extensive subthalamic oscillatory synchronization. Neuroscience, 171, 245-257.

Ray, S. \& Maunsell, J.H. (2011) Different origins of gamma rhythm and high-gamma activity in macaque visual cortex. PLoS Biol., 9, e1000610.

Rossi, L., Marceglia, S., Foffani, G., Cogiamanian, F., Tamma, F., Rampini, P., Barbieri, S., Bracchi, F. \& Priori, A. (2008) Subthalamic local field potential oscillations during ongoing deep brain stimulation in Parkinson's disease. Brain Res. Bull., 76, 512-521.

Sederberg, P.B., Schulze-Bonhage, A., Madsen, J.R., Bromfield, E.B., McCarthy, D.C., Brandt, A., Tully, M.S. \& Kahana, M.J. (2007) Hippocampal and neocortical gamma oscillations predict memory formation in humans. Cereb. Cortex, 17, 1190-1196.

Sirota, A., Montgomery, S., Fujisawa, S., Isomura, Y., Zugaro, M. \& Buzsáki, G. (2008) Entrainment of neocortical neurons and gamma oscillations by the hippocampal theta rhythm. Neuron, 60, 683-697.

Stam, C.J., van Cappellen van Walsum A.M., Pijnenburg, Y.A., Berendse, H.W., de Munck, J.C., Scheltens, P. \& Van Dijk, B.W. (2002) Generalized synchronization of MEG 
recordings in Alzheimer's Disease: Evidence for involvement of the gamma band. J. Clin. Neurophysiol., 19, 562-574.

Swann, N., Poizner, H., Houser, M., Gould, S., Greenhouse, I., Cai, W., Strunk, J., George, J. \& Aron, A.R. (2011) Deep brain stimulation of the subthalamic nucleus alters the cortical profile of response inhibition in the beta frequency band: A scalp EEG study in Parkinson's disease. J. Neurosci., 31, 5721-5729.

Tass, P.A., Silchenko, A.N., Hauptmann, C., Barnikol, U.B. \& Speckmann, E.J. (2009) Longlasting desynchronization in rat hippocampal slice induced by coordinated reset stimulation. Phys. Rev. E, 80, 011902.

Thompson, J.A., Lanctin, D., Ince, N.F. \& Abosch, A. (2014) Clinical implications of local field potentials for understanding and treating movement disorders. Stereotact. Funct. Neurosurg., 92, 251-263.

Uhlhaas, P.J. \& Singer, W. (2006) Neural synchrony in brain disorders: Relevance for cognitive dysfunctions and pathophysiology. Neuron, 52, 155-168.

Vianney-Rodrigues, P., Iancu, O.D. \& Welsh, J.P. (2011) Gamma oscillations in the auditory cortex of awake rats. Eur. J. Neurosci., 33, 119-129.

Wang, S.Y., Aziz, T.Z., Stein, J.F. \& Liu, X. (2005) Time-frequency analysis of transient neuromuscular events: Dynamic changes in activity of the subthalamic nucleus and forearm muscles related to the intermittent resting tremor. J. Neurosci. Methods, 145, 151158.

Wasterlain, C.G., Shirasaka, Y., Mazarati, A.M. \& Spigelman, I. (1996) Chronic epilepsy with damage restricted to the hippocampus: Possible mechanisms. Epilepsy Res., 26, 255265.

Wingeier, B., Tcheng, T., Koop, M.M., Hill, B.C., Heit, G. \& Bronte-Stewart, H.M. (2006) Intra-operative STN DBS attenuates the prominent beta rhythm in the STN in Parkinson's disease. Exp. Neurol., 197, 244-251.

Zaveri, H.P., Williams, W.J., Sackellares, J.C., Beydoun, A., Duckrow, R.B. \& Spencer, S.S. (1999) Measuring the coherence of intracranial electroencephalograms. Clin. Neurophysiol., 110, 1717-1725. 